\title{
Les accents ecclésiologiques du pontificat du pape François. Une mise en œuvre originale de Lumen gentium ${ }^{7}$
}

\author{
The ecclesiological accents of the pontificate of Pope \\ Francis. An original reception of Lumen Gentium
}

Gilles Routhier

\section{Resumo}

O que Jorgje Bergolio conheceu como jovem religioso e padre, é uma Igreja renovada pelo Concílio Vaticano II. Para ele, o Vaticano II é algo de natural, que ele integrou. Além disso, ele não toma o seu tempo para discutir, ele « faz » e « fala »Vaticano II. Sua ação é linguagem performativa. Ainda, fiel à eclesiologia da Lumen Gentium, ele considera a Igreja, povo de Deus em marcha, como sujeito na história. Ele também toma os membros da Igreja, bispos (sozinhos ou reunidos em Conferências Episcopais), pastores, fiéis leigos, como sujeitos de ação e de iniciativas na Igreja. Ele os reconhece como sujeitos de ação e autoriza a sua iniciativa. Este artigo mostra, a partir de uma leitura das exortações apostólicas Evangelii Gaudium e Amoris Laetitia, como Francisco constitui as Igrejas locais e seus membros como sujeitos de ação e de iniciativas, atualizando assim a eclesiologia do Vaticano II.

Palavras-chave: Papa Francisco. Eclesiologia do Vaticano II. Evangelii Gaudium. Igreja como sujeito de sujeitos.

\footnotetext{
${ }^{1}$ Este artigo corresponde à apresentação que o Prof. Gilles Routhier proferiu, no dia 19 de setembro, junto ao Congresso Internacional de Teología «Interpelaciones del Francisco a la Teología hoy», realizado de 18 a 21 de setembro de 2016 na Pontificia Universidad Javeriana.
} 


\title{
Résumé
}

Ce qu'a connu Jorge Bergolio comme jeune religieux et prêtre, c'est une Église rénovée par le concile Vatican II. Vatican II est pour lui un allant de soi quelque chose qui lui est naturel, qu'il a intégré. Aussi, il ne passe pas tout son temps à en discuter, il « fait » et il «parle » Vatican II. Son action est langage performatif. Aussi, fidèle à l'ecclésiologie de Lumen gentium, il considère l'Église, peuple de Dieu en marche, comme sujet dans l'histoire. Il rend aussi les membres de l'Église, évêques (seuls ou réunis en conférences épiscopales), pasteurs, fidèles laïcs, sujets d'actions et d'initiatives dans l'Église. Il les reconnaît comme sujets d'action et autorise leur initiative. Le présent article montre, à partir d'une lecture des exhortations apostoliques Evangelii gaudium et Amoris laetitia, comment François constitue les Églises locales et leurs membres comme sujets d'actions et d'initiatives, actualisant ainsi l'ecclésiologie de Vatican II.

Mots clés: Pape François. Ecclésiologie de Vatican II. Evangelii gaudium. Église sujet et de sujets.

\begin{abstract}
What Jorge Bergolio has known as a young religious and priest is a Church renewed by the Second Vatican Council. For him, Vatican II is something natural, which he has integrated. Therefore, he does not spend all of his time discussing it, he «does» and «speaks» Vatican II. His action is performative language. Hence, true to the ecclesiology of Lumen Gentium, he considers the Church, the wandering people of God, as a subject of history. He also makes of the members of the Church, bishops (alone or together within Episcopal Conferences), pastors or lay faithful, subjects of action and initiative in the Church. He recognizes them as subjects of action and authorizes their initiative. This article shows, from a reading of the apostolic exhortations Evangelii gaudium and Amoris laetitia, how Francis constitutes the local Churches and their members as subjects of action and initiative, thus implementing, in a way consistent with the needs of the present time, the ecclesiology of Vatican II.
\end{abstract}

Keywords: Pope Francis. Ecclesiology of Vatican II. Evangelii gaudium. Church as a subjet of subjects. 


\section{Introduction}

Les historiens des conciles nous enseignent que toute périodisation de la réception d'un concile doit considérer comme un repère la disparition de la génération qui a fait le concile. G. Alberigo, par exemple, sur la base de ses observations de la réception de Trente et de Vatican II, note qu'une phase importante de la réception s'achève lorsque les participants à un concile (évêques et théologiens) cessent d'être les protagonistes de la vie ecclésiale ${ }^{2}$. Pour lui, la période qui suit immédiatement un concile risque d'être déterminée par la projection, dans la vie ecclésiale, des débats, des tensions, voire des oppositions, qui traversaient l'assemblée conciliaire. Suivant ce critère, l'élection de François signerait le passage à une nouvelle phase de la réception de Vatican II. Entré au noviciat en 1958, Jorge Bergolio a vécu les premières années de sa vie religieuse dans une Église marquée par le pontificat de Roncalli et l'atmosphère joyeux et évangélique de la période préconciliaire et des premiers pas du concile Vatican II. Plus important encore, sa formation théologique (1967-1970) a bénéficié du renouveau de la théologie mis en avant par le Concile Vatican II. Ordonné prêtre en 1969, l'Église qu'il a connu, c'est une Église conciliaire.

Depuis son élection, François n'a pas fait de grands discours sur Vatican II. Par-dessus tout, il s'est bien gardé de relancer le débat sur l'herméneutique de Vatican II. Il s'est tenu à distance de ces discussions oiseuses et stériles qui ne font pas avancer les choses. Plutôt que de parler de Vatican II, il «parle Vatican II» à travers toute son action. En effet, par son attitude, par ses options et par son action, il «fait Vatican II». On pourrait dire, inversant les termes du titre du philosophe du langage J.L. Austin, que pour lui, faire, c'est dire ${ }^{3}$. Ses actions sont langage performatif. Aussi, il ne discourt pas sur Vatican II, mais son discours et son toute action disent et enseignent Vatican II et sont à la manière de Vatican II ou selon son style.

À titre d'exemples, son exhortation apostolique Evangelii gaudium ne comporte, sur les 217 notes que compte le document, que 17 renvois explicites à un passage ou à un document de Vatican II. L'examen de chacune d'eux nous montre que, au final, ces renvois ne sont pas déterminants dans

${ }^{2} C f$. « La condition chrétienne après Vatican II », dans La réception de Vatican II, dans Giuseppe Alberigo et Jean-Pierre Jossua (dir.). La réception de Vatican II. Paris: Cerf, 1985, sp. pp. 12-19.

${ }^{3}$ John Langshaw Austin. Quand dire, c'est faire. Paris: Seuil, 1970. 
l'argumentation et qu'ils sont plus souvent qu'autrement décoratifs. Par ailleurs, l'examen de ces notes nous révèle d'autres surprises. C'est ainsi que l'on trouve 22 références à des documents de conférences épiscopales (des États-Unis, de France, du Brésil, des Philippines, de l'Inde), ou d'assemblées régionales d'évêques (en particulier du CELAM, mais aussi de l'Europe).

C'est ainsi que, dans son Exhortation apostolique Evangelii gaudium, plutôt que de faire un long discours sur la collégialité épiscopale - on n'y trouve qu'un bref mais décisif développement ( $\left.\mathrm{n}^{\circ} 32\right)$ avec une citation d'une demi-phrase (8 mots) de Lumen gentium 23 - il valorise concrètement les conférences épiscopales et les regroupements continentaux d'évêques. Cela n'est pas une exception, car il répète le même geste dans son encyclique Laudato $\mathrm{Si}$, dans laquelle, en plus d'assumer de manière œcuménique l'enseignement du Patriarche Bartholomé, il cite la FABC et le CELAM et des documents des conférences épiscopales de l'Afrique du Sud, de l'Allemagne, de l'Argentine, de l'Australie, du Brésil, de la Bolivie, du Canada, des ÉtatsUnis, du Japon, de la Nouvelle-Zélande, du Mexique, du Paraguay, des Philippines, du Portugal, de la République dominicaine. Quant à l'Exhortation apostolique Amoris laetetia, on y trouve seize renvois à Vatican II, surtout la Constitution pastorale Gaudium et spes, et neuf aux conférences épiscopales nationales (Espagne, Corée, Argentine, Mexique, Colombie, Chili, Australe, Italie et Kenya) et un à la Conférence générale de l'épiscopat latino-américain d'Aparecida. Il assume donc le magistère des conférences épiscopales ou des regroupements continentaux d'évêques, sa parole est liée à la leur.

Cela est sans précédent et ce ne peut être fortuit. Il s'agit sans doute d'un geste volontaire qui exprime une intention. François ne se contente pas d'observer que le souhait formulé par Vatican II visant un exercice plus collégial du gouvernement de l'Église catholique «ne s'est pas pleinement réalisé, parce que n'a pas encore été suffisamment explicité un statut des conférences épiscopales qui les conçoive comme sujet d'attributions concrètes, y compris une certaine autorité doctrinale authentique» ( $E g$ 32). Il offre, de manière très concrète, une réalisation de ce vœu. C'est en ce sens que je dis qu'il «fait Vatican II» ou qu'il «parle Vatican II». Il n'ouvre pas un nouveau débat sur l'autorité doctrinale des conférences épiscopales. Il la reconnaît et il l'autorise.

Je crois qu'il s'agit là des deux verbes les plus importants, s'agissant de caractériser la contribution du pape François à la réception de l'ecclésiologie de Vatican II : il reconnaît des sujets d'action et d'initiatives et il autorise des 
personnes et des groupes à être sujets aptes à participer activement à la vie de l'Église comprise comme peuple de Dieu. Il ne fait pas du pape l'unique sujet d'action et il ne monopolise pas tout l'espace d'initiative, lui-même se présentant fréquemment à partir de son titre d'Évêque de Rome. Il permet à d'autres sujets d'exister et les encourage à prendre des initiatives et à jouer pleinement le rôle qui leur revient dans l'Église. En particulier, il autorise les conférences épiscopales - on l'a déjà vu -, plus spécifiquement les évêques et les pasteurs et les fidèles laïcs. De manière globale, il autorise les Églises locales, j'aurais dû commencer par-là, comme le fait Lumen gentium dans son chapitre II, qui met en premier lieu l'Église, avant de traiter de ses divers membres. D'ailleurs, le pape François assume cet enseignement de Vatican II. On en trouve un témoignage dans sa lettre du 9 mars dernier au cardinal Marc Ouellet, président de la Commission sur l'Amérique latine : "Parler du saint peuple de Dieu, c'est parler de l'horizon vers lequel nous sommes appelés à regarder et dont notre réflexion doit partir. »

\section{Autoriser les Églises locales et les communautés à être sujets actifs}

François, citant son prédécesseur, reprend un jugement que Paul VI formulait dans sa lettre apostolique Octogesima adveniens qui affirmait que

« Face à des situations aussi variées, il nous est difficile de prononcer une parole unique, comme de proposer une solution qui ait une valeur universelle. Telle n'est pas notre ambition, ni même notre mission. Il revient aux communautés chrétiennes d'analyser avec objectivité la situation propre de leur pays ». (Oa 4, repris dans $E g$ 184).

Paul VI reconnaissait les communautés chrétiennes qu'il invitait à analyser les situations propres de leur pays. François ne fait pas autrement lorsqu'il déclare : «Ce n'est pas la tâche du Pape de présenter une analyse détaillée et complète de la réalité contemporaine, mais j'exhorte toutes les communautés à avoir « l'attention constamment éveillée aux signes des temps». ( $E g$ 51).

Dans le cas, ce sont les communautés qu'il reconnaît comme sujets d'action et d'initiative et qu'il autorise à lire les signes des temps, comme le faisait Vatican II, et qu'il autorise à conduire un discernement, mot qui revient fréquemment sous la plume du pape (31 fois dans Amoris laetetia) : «Tout chrétien et toute communauté discernera quel est le chemin que le Seigneur 
demande, mais nous sommes tous invités à accepter cet appel : sortir de son propre confort et avoir le courage de rejoindre toutes les périphéries qui ont besoin de la lumière de l'Évangile.» (Eg 20). Ce sont aussi les Églises locales qu'il établit comme sujet, «sujet premier de l'évangélisation», écrit-il, rappelant la phrase si commentée de Vatican II à savoir «qu'en elle 'est vraiment présente et agissante l'Église du Christ, une, sainte, catholique et apostolique'». Ces Églises locales, il les considère comme sujet de discernement : «Pour que cette impulsion missionnaire soit toujours plus intense, généreuse et féconde, j'exhorte aussi chaque Église particulière à entrer dans un processus résolu de discernement, de purification et de réforme.» ( $E g$ 30) Ce discernement, laissé aux Églises locales, concerne les chemins d'évangélisation, les appels à la conversion, mais également les actions à prendre pour accompagner les couples et les familles : «Il y a diverses manières légitimes d'organiser la préparation immédiate au mariage, et chaque Église locale discernera ce qui est mieux, en offrant une formation adéquate qui en même temps n'éloigne pas les jeunes du sacrement.» (Al 207)

Ayant identifié les Églises locales comme premier sujet de discernement des voies à prendre pour l'annonce de l'Évangile ou des moyens à prendre pour faire face à des situations complexes, François décline par la suite les responsabilités de chacun, celle des pasteurs, les évêques, pris individuellement ou réunis en conférences épiscopales, et les prêtres, mais également celle des fidèles-laïcs. Fait à noter, il n'isole pas chaque personne, mais les situe dans l'Église et, souvent, il indique la coopération qu'ils doivent entretenir les uns avec les autres.

\section{Autoriser les épiscopats locaux}

C'est en ce sens, qu'il exprime clairement et à trois reprises qu'on ne doit pas «attendre du magistère papal une parole définitive ou complète sur toutes les questions qui concernent l'Église et le monde. Il n'est pas opportun que le Pape remplace les Épiscopats locaux dans le discernement de toutes les problématiques qui se présentent sur leurs territoires. En ce sens, je sens la nécessité de progresser dans une "décentralisation" salutaire» $\left(\mathrm{n}^{\circ} 16\right)$.

Ailleurs, citant la relatio finalis et ayant en vue des situations matrimoniales complexes, le pape écrit: "'Dans ces cas-là, les évêques sont appelés à exercer un discernement pastoral adapté à leur bien spirituel'». ( $\mathrm{Al}$ 249) Alors que Paul VI, sur les questions sociales, reconnaissait faire face à des «situations variées» qui rendait «difficile de prononcer une parole unique» 
ou «de proposer une solution qui ait une valeur universelle», François élargit le champ et reconnaît pour sa part faire face à la même difficulté lorsqu'il s'agit de traiter des questions familiales. Dans ce domaine aussi, les situations sont diverses et complexes. Il adapte ainsi la déclaration de Paul VI:

«Si l'on tient compte de l'innombrable diversité des situations concrètes, comme celles mentionnées auparavant, on peut comprendre qu'on ne devait pas attendre du Synode ou de cette Exhortation une nouvelle législation générale du genre canonique, applicable à tous les cas. Il faut seulement un nouvel encouragement au discernement responsable personnel et pastoral des cas particuliers ${ }^{4} . \gg($ Al 300)

Cela le conduit à reconnaître aux pasteurs la nécessité de discerner plutôt que d'attendre une solution universelle venant de la parole unique du pape. Aussi, les invite-t-il, non seulement à faire la promotion du mariage chrétien, mais aussi à engager un

«'discernement pastoral des situations de beaucoup de gens qui ne vivent plus dans cette situation' pour 'entrer en dialogue pastoral avec ces personnes afin de mettre en évidence les éléments de leur vie qui peuvent conduire à une plus grande ouverture à l'Évangile du mariage dans sa plénitude'». (Al 293)

\section{Autoriser les fidèles-laïcs}

Ayant autorisé les communautés et les Églises locales, les évêques, les conférences épiscopales et les pasteurs, en les reconnaissant comme sujet d'initiative dans l'Église, il autorise également tous les baptisés qui sont appelés, eux aussi, non seulement à travailler à la vigne du Seigneur, mais également à prendre part au discernement ecclésial qui conduit à des prises de décision. Ce droit se fonde sur le sensus fidelium, charisme qui appartient à tous les fidèles : «Le Peuple de Dieu est saint à cause de cette onction que le rend infaillible "in credendo". Cela signifie que quand il croit il ne se trompe pas, même s'il ne trouve pas les paroles pour exprimer sa foi ${ }^{5}$. L'Esprit le

\footnotetext{
${ }^{4}$ Voir aussi le $\mathrm{n}^{\mathrm{o}} 304$ où il rappelle l'enseignement de Thomas d'Aquin à ce sujet.

${ }^{5}$ Sur le Sensus fidei, voir Gilles Routhier, «Le Sensus fidei. L'interrelation entre tous les membres du peuple de Dieu» dans Timothy Radcliffe et Gilles Routhier Vox populi, vox Dei?. Paris: Médiaspaul, 2016, pp. 41-76.
} 
guide dans la vérité et le conduit au salut. [...] Dieu dote la totalité des fidèles d'un instinct de la foi - le sensus fidei - qui les aide à discerner ce qui vient réellement de Dieu. » $\left(\mathrm{Eg} 119^{6}\right)$. De fait, François revient très fréquemment sur le sensus fidei, autre enseignement de Lumen gentium (voir le $\mathrm{n}^{\circ} 12$ ). Cela est déjà présent en filigrane dans ce passage de son premier Angélus alors que François évoque, à travers un récit, l'instinct de la foi ou le flair spirituel d'une vieille dame : «Est venue à moi une femme âgée, humble, très humble, elle avait plus de quatre-vingts ans. Je l'ai regardée et je lui ai dit : " Grand-mère [...] vous voulez vous confesser ?». " Oui ! », m'a-t-elle dit. «Mais si vous n'avez pas péché...». Et elle m’a dit : «Nous avons tous péché... !». « Mais peut-être le Seigneur ne les pardonne pas... ». « Le Seigneur pardonne tout !», m'a-t-elle dit : sûre d'elle. " Mais comment le savez-vous, vous, Madame? ». « Si le Seigneur ne pardonnait pas tout, le monde n'existerait pas ». Il m'est venue l'envie de lui demander : « Dites-moi, Madame, vous avez étudié à la Grégorienne ? », parce que cela est la sagesse que donne l'Esprit Saint ; la sagesse intérieure vers la miséricorde de Dieu.» (Angélus, 17 mars 2013) Il y revient à nouveau dans son entrevue avec le P. Sparado de la Civilta cattolicà, parlant du sentire cum Ecclesia.

C'est sans doute la confiance à ce sens de la foi qui l'a amené à suggérer que les fidèles des diocèses soient consultés au cours de la préparation des assemblées du Synode des évêques sur la famille, articulant ainsi la collégialité des évêques à la synodalité de toute l'Église. Ici encore, il reconnaît aux fidèles la capacité de discerner.

De fait, il reconnaît que tous les baptisés sont sujets actifs, «disciplesmissionnaires»:

En vertu du Baptême reçu, chaque membre du Peuple de Dieu est devenu disciple missionnaire (cf. Mt 28, 19). Chaque baptisé, quelle que soit sa fonction dans l'Église et le niveau d'instruction de sa foi, est un sujet actif de l'évangélisation, et il serait inadéquat de penser à un schéma d'évangélisation utilisé pour des acteurs qualifiés, où le reste du peuple fidèle serait seulement destiné à bénéficier de leurs actions. La nouvelle évangélisation doit impliquer que chaque baptisé soit protagoniste d'une façon nouvelle.

\footnotetext{
${ }^{6}$ Ce passage est précédé d'un enseignement sur l'infaillibilité du peuple de Dieu in credendo. Sur le sensus fidei, voir aussi les $\mathrm{n}^{\text {os }} 198$.
} 
Il rompt avec les schèmes pastoraux qui font des uns des sujets d'action et des autres les bénéficiaires de l'action des pasteurs.

Plus spécifiquement, il veut instituer les fidèles-laïcs comme sujets actifs ou protagonistes de la vie ecclésiale et de l'évangélisation. Dans son discours d'ouverture de l'assemblée générale de la CEI (18 mai 2015), il demande aux évêques de permettre cette avènement : «La sensibilità ecclesiale e pastorale si concretizza anche nel rinforzare l'indispensabile ruolo di laici disposti ad assumersi le responsabilità che a loro competono. In realtà, i laici che hanno una formazione cristiana autentica, non dovrebbero aver bisogno del Vescovopilota, o del monsignore-pilota o di un input clericale per assumersi le proprie responsabilità a tutti i livelli, da quello politico a quello sociale, da quello economico a quello legislativo! Hanno invece tutti la necessità del Vescovo Pastore!»

Constamment, il dénonce le mal du cléricalisme. Il le fait de manière éloquente dans sa lettre du 9 mars dernier au cardinal Ouellet ${ }^{7}$. Il y articule également le lien entre pasteurs et fidèles laïcs : « Le pasteur est pasteur d'un peuple, et ce peuple, il doit le servir de l'intérieur. Le plus souvent on avance en ouvrant le chemin, mais d'autres fois il nous faut revenir sur nos pas pour que personne ne reste en arrière, et il n'est pas rare de devoir rester au milieu pour bien entendre le cœur des gens. »

\section{Articuler les initiatives des pasteurs et celle des laïcs}

Cette valorisation de la capacité des fidèles laïcs à discerner reviendra constamment dans son Exhortation apostolique Amoris laetitia. Toutefois, il n'isole pas un sujet (les pasteurs ou les fidèles laïcs), mais il est toujours soucieux de présenter la coopération entre les divers sujets, en particulier en articulant l'action des pasteurs et celle des laïcs. Il note d'abord la difficulté qu'ont les

\footnotetext{
${ }^{7}$ Dans cette lettre, il écrivait ces lignes : « Non seulement cette attitude annule la personnalité du chrétien, mais elle tend à diminuer et à sous-estimer la grâce baptismale que l'Esprit Saint a mise dans le cœur de nos fidèles. Le cléricalisme porte à une uniformisation du laïcat ; en le traitant comme un simple «mandataire », il limite toutes les initiatives et tous les efforts, et j'oserai dire les audaces nécessaires, pour porter la bonne nouvelle de l'Evangile à tous les domaines de l'activité sociale et surtout politique. Le cléricalisme, loin de donner de l'impulsion aux différentes contributions et propositions, éteint peu à peu le feu prophétique dont l'Eglise entière est appelée à témoigner dans le cœur de ses peuples. Le cléricalisme oublie que la visibilité et la sacramentalité de l'Eglise appartiennent à tout le peuple de Dieu (cf. Lumen gentium, n. 9-14), et non à une seule poignée d'élus et de personnes éclairées. »
} 
pasteurs à faire confiance aux fidèles, à leur jugement et à leur discernement : «Il nous coûte aussi de laisser de la place à la conscience des fidèles qui souvent répondent de leur mieux à l'Évangile avec leur limites et peuvent exercer leur propre discernement dans des situations où tous les schémas sont battus en brèche. Nous sommes appelés à former les consciences, mais non à prétendre nous substituer à elles. » ( $\mathrm{Al} 37$ ) Ici, il articule la coopération entre les pasteurs et les fidèles laïcs. À plusieurs reprises, s'appuyant sur l'enseignement de Gaudium et spes, François revient sur le respect de la conscience des fidèles et sur la capacité de discernement des couples ${ }^{8}$. Certes, l'appel à l'autorité de la conscience engage la formation de la conscience qui présuppose la coopération entre pasteurs et fidèles dans le processus de discernement qui est engagé : « Évidemment, il faut encourager la maturation d'une conscience éclairée, formée et accompagnée par le discernement responsable et sérieux du Pasteur, et proposer une confiance toujours plus grande dans la grâce. (Al 303). On est en présence d'une conspiration (conspiratio) entre laïcs et pasteurs, ce qui est également dans le droit fil de l'ecclésiologie de Vatican II $^{9}$. Dans son entrevue à son confrère jésuite de la Civilta cattolicà, il dira : le «Sentire cum Ecclesia, c'est pour moi être au milieu de ce peuple.» L'infaillibilitas in credendo, ajoute-t-il, «c'est l'expérience [...] de l'Église comme peuple de Dieu, pasteurs et peuple tous ensemble.» C'est donc en vivant au milieu de ce peuple et c'est en aimant ce peuple, jusqu'à sentir l'odeur des brebis, que les évêques et les prêtres apprennent de ce peuple, des pauvres surtout. C'est dans ce contexte que le pape François fait intervenir le sensus fidei : «Pour cette raison, je désire une Église pauvre pour les pauvres. Ils ont beaucoup à nous enseigner. En plus de participer au sensus fidei, par leurs propres souffrances ils connaissent le Christ souffrant. Il est nécessaire que tous nous nous laissions évangéliser par eux.» (Eg 198)

\footnotetext{
${ }^{8}$ « Ce que le Concile Vatican II a exprimé avec clarté est encore valable : 'D’un commun accord et d'un commun effort, [les époux] se formeront un jugement droit : ils prendront en considération à la fois et leur bien et celui des enfants déjà nés ou à naître ; ils discerneront les conditions aussi bien matérielles que spirituelles de leur époque et de leur situation; ils tiendront compte enfin du bien de la communauté familiale, des besoins de la société temporelle et de l'Église elle-même. Ce jugement, ce sont en dernier ressort les époux eux-mêmes qui doivent l'arrêter devant Dieu' ». (Al 222)

${ }^{9}$ Sur la tâche de discernement des pasteurs, voir le $n^{\circ} 79$ (« Face aux situations difficiles et aux familles blessées, il faut toujours rappeler un principe général : "Les pasteurs doivent savoir que, par amour de la vérité, ils ont l'obligation de bien discerner les diverses situations" (Familiaris consortio, n. 84). »)
} 
Les prêtres ont la mission « d'accompagner les personnes intéressées sur la voie du discernement selon l'enseignement de l'Église et les orientations de l'évêque. » $\left(A l 300^{10}\right)$ Cette coopération est rappelée au no 297 d'Al : il revient au conjoint divorcé et réengagé de mener un discernement et de « suggérer », " de sa propre initiative », la « manière propre », « en accord avec le discernement du Pasteur », la manière suivant laquelle il entend participer à la vie de l’Église.

\section{Conclusion}

Voilà ce que j'ai pu relever des accents ecclésiologiques du pontificat de François en faisant une lecture des Exhortations apostoliques Evangelii gaudium et Amoris laetetia, pour me limiter à ces deux textes importants ${ }^{11}$. Peut-on conclure à partir de là que nous sommes entrés dans une nouvelle phase de la réception de Vatican II ou s'il ne s'agit que d'une simple parenthèse dans ce processus encore ouvert et de longue portée? Il est sans doute trop tôt pour le dire. De plus, il faudrait élargir l'enquête à l'ensemble du pontificat et examiner non seulement ses écrits, mais également ses gestes. Il faudrait surtout examiner si cette attitude et ces gestes se traduisent dans des textes législatifs et s'incarnent dans des institutions, ce qui est une autre question.

Je l'ai fait cet exercice en insistant sur la catégorie de « sujet». Je ne l'ai pas importé d'une théorie ecclésiologique avant de la projeter ensuite dans le discours du pape François. Je l'ai emprunté à son propre discours ${ }^{12}$. En

\footnotetext{
${ }^{10} \mathrm{Au}$ même numéro, il précisera cette démarche engageant pasteur et laïc : « Il s'agit d'un itinéraire d'accompagnement et de discernement qui « oriente ces fidèles à la prise de conscience de leur situation devant Dieu. Le colloque avec le prêtre, dans le for interne, concourt à la formation d'un jugement correct sur ce qui entrave la possibilité d'une participation plus entière à la vie de l'Église et sur les étapes à accomplir pour la favoriser et la faire grandir. » Voir aussi le $n^{\circ} 303$ : « il faut encourager la maturation d'une conscience éclairée, formée et accompagnée par le discernement responsable et sérieux du Pasteur. »

${ }^{11}$ On verra aussi Christoph Theobald, « L'Exhortation apostolique Evangelii gaudium. Esquisse d'une interprétation originale du Concile Vatican II », dans Revue théologique de Louvain, 46 (2015), pp. 321-340.

${ }^{12}$ Quelques ecclésiologues ont mis en avant cette conceptualité. Voir Yves Congar, ««L'ecclesia» ou communauté chrétienne, sujet intégral de l'action liturgique ", in Yves Congar et JeanPierre Jossua, dir., La liturgie après Vatican II. Paris: Cerf, 1967, pp. 241-282; Hervé Legrand, " Le développement d'Églises-sujets une requête de Vatican II », in Giuseppe Alberigo dir., Les Églises après Vatican II: dynamisme et prospective. Paris: Beauchesnes, coll. Théologie historique 61, 1981, pp. 149-184; Gustave Thils, « La communauté ecclésiale sujet d'action et sujet de droit ». Revue Théologique de Louvain 4 (1973), pp. 443-468.
} 
effet, la catégorie de «sujet» revient souvent sous sa plume : L'Église locale est «est le sujet premier de l'évangélisation» $\left(E g \mathrm{n}^{\circ} 30\right)$, comme l'est du reste l’Église toute entière, le peuple de Dieu («ce sujet de l'évangélisation est bien plus qu'une institution organique et hiérarchique, car avant tout c'est un peuple qui est en marche vers Dieu ${ }^{13} . n^{\circ} 111$ ), comme le sont aussi «les divers peuples, chez qui l'Évangile a été inculturé, [qui] sont des sujets collectifs actifs, agents de l'évangélisation.» ( $\left.{ }^{\circ} 122\right)$ les conférences épiscopales, qu'il regrette de ne pas les voir conçues «comme sujet d'attributions concrètes» $\left(\mathrm{n}^{\circ}\right.$ 32) En somme, tout au long de l'Exhortation apostolique Evangelii gaudium, on voit des personnes ou des groupes être constitués comme sujets d'action, reconnues comme sujets d'initiative.

J'aurais pu mettre en évidence certains autres aspects qui présentent des similitudes entre son programme de pontificat formulé dans Evangelii gaudium et le Concile Vatican II. D'une part, il y a la connexion étroite qu'il établit entre évangélisation et réforme, lien qui s'élargit en rendant encore plus explicite une intuition du concile qui est demeuré sous-développée, soit le lien entre les pauvres, l'évangélisation et la réforme. Au plan méthodologique, j'aurais pu relever également son attachement aux realia, tout le chapitre II d'Amoris laetetia intitulé «La réalité et les défis de la famille » y étant consacré. Renvoyant à l'enseignement de Jean-Paul II, François rappelle qu' « Il convient de prêter attention à la réalité concrète, parce que « les exigences, les appels de l'Esprit se font entendre aussi à travers les événements de l'histoire » $\left(\mathrm{n}^{\circ} 31\right)$ Cela nous rapproche de l'option prise par les Pères conciliaires au moment de rédiger Gaudium et spes. De même, son attention à la culture et à l'inculturation, catégorie mise en avant par le P. Aruppe. L'Église et l'Évangile sont inscrits dans des cultures, ce qui le rapproche également de l'ecclésiologie mise en avant par Vatican II.

Il faudrait examiner d'autres aspects de son pontificat. Parmi ceuxci, une attention particulière devrait être portée au volet œcuménique. Ordonné prêtre après Vatican II, il a intégré depuis plusieurs années la dimension œcuménique à son ministère. Cela est pour lui naturel et son attitude n'est manifestement pas apprise ou composée. Il en est de même pour les rapports interreligieux. Les apprentissages se sont faits tout au

\footnotetext{
${ }^{13}$ Voir aussi le $\mathrm{n}^{\circ} 129$ : «le peuple de Dieu, avec ses innombrables gestes et signes, est le sujet collectif.» Ailleurs, au sujet du processus de pacification : «L'auteur principal, le sujet historique de ce processus, c'est le peuple et sa culture, et non une classe, une fraction, un groupe, une élite.» ( $\left.n^{\circ} 229\right)$
} 
long de sa vie, à travers des rencontres avec des personnes. Dans tous les cas, ce qui est mis en avant, ce sont les personnes et les liens personnels et non la doctrine ou les discussions doctrinales. Cela nous amène à dire que, comme à Vatican II, le style pastoral (ou style évangélisateur ou missionnaire comme il le développera aux $\mathrm{n}^{\text {os }} 18,27,33,35$ et 75 d'Evangelii gaudium), plus que la régulation théologique, est mis à la première place. De même, il va au «cœur de l'Évangile ${ }^{14}$ » et il a intégré le critère de la hiérarchie des vérités mis en avant par Vatican II. Ce rappel de Vatican II ne représente pas une citation décorative de Evangelii gaudium $\left(\mathrm{n}^{\mathrm{o}} 36\right)$, mais elle est structurante, non seulement de ce passage, mais également son développement sur l'homélie.

N'appartenant pas au monde européen, malgré les racines italiennes de sa famille et le caractère européen de Buenos Aires, il est plus naturellement attentif aux culture ${ }^{15}$ du monde, comme l'a été Vatican II, premier concile d'une Église aux dimensions du monde. Il voit également l'économie mondiale à partir d'un autre point de vue que celui dominant dans les pays du Nord.

En somme, probablement que, comme Vatican II, c'est d'abord par son style, style de vie et style pastora ${ }^{16}$, qu'il marque l'Église. Ensuite, comme Vatican II, c'est dans son rapport aux autres, rapport aux personnes, d'abord, personnes à qui il accorde priorité, rapport au monde, aux pauvres, aux cultures, aux non croyants, aux croyants non chrétiens, aux chrétiens non catholiques, etc. Cela n'est pas sans rappeler le caractère excentré ou non autoréférentiel du concile Vatican II dont l'enseignement se ramène aux relations : relation à Dieu (dialogue de la Révélation et de la liturgie), relation avec le monde (Gaudium et spes), relation avec les chrétiens non-catholiques (Unitatis redintegratio), relation avec les non-chrétiens (Nostra Atate et Ad gentes). C'est en troisième lieu, par son enseignement, enseignement libre, authentique, qui fait autorité en raison même de sa liberté et de son authenticité. C'est encore en raison de l'attention qu'il porte au peuple de Dieu et à une conception décentralisée de l'Église.

\footnotetext{
${ }^{14}$ C'est ainsi qu'il intitule la section 3 du chapitre I : «À partir du cœur de l'Évangile». ${ }^{15}$ Voir en particulier les nos 30 et 41.

${ }^{16}$ Le mot style revient à 24 reprises dans l'Exhortation apostolique, du moins dans l'édition française, onze fois pour parler du style de vie, mais plusieurs fois pour évoquer les style pastoral, notamment lorsqu'il parle du style de Jésus, son style de vie et son style pastoral, les deux étant liés.
} 
Enfin, son pontificat rappelle Vatican II en raison des décisions structurantes qu'il prend, ce qui engage le droit. S'il n'allait pas jusque-là, le souffle rénovateur qu'il donne actuellement à l'Église risquerait d'être sans lendemain. Sans doute, cela n'est pas premier, l'impulsion est avant tout spirituelle, mais on ne peut en faire l'économie. Sans doute compte-t-il aussi que ce sont les Églises locales qui sont appelées à prendre le relai, car, au final, ce sont elles qui sont les premiers sujets de l'évangélisation et, à ce titre, elles sont appelées à «entrer dans un processus résolu de discernement, de purification et de réforme» ( $E g$ 30). De plus, c'est le peuple de Dieu tout entier, le plérôme de l'Église, qui doit être rénové. Encore une fois, le pape ne peut pas tout et la nouvelle phase de la réception de Vatican II appartient aux Églises locales et au corps tout entier de l'Église.

\section{Références bibliographiques}

Alberigo, Giuseppe. " La condition chrétienne après Vatican II », dans La réception de Vatican II, dans Giuseppe Alberigo et Jean-Pierre JossuA (dir.). La réception de Vatican II. Paris: Cerf, 1985, sp. pp. 12-19.

Austin, John Langshaw. Quand dire, c'est faire. Paris: Seuil, 1970.

Congar, Yves. « «L〉ecclesia» ou communauté chrétienne, sujet intégral de 1)action liturgique », in Yves Congar et Jean-Pierre Jossua, dir. La liturgie après Vatican II. Paris: Cerf, 1967, pp. 241-282.

Françors, Exhortation apostolique Evangelii gaudium, Rome, 24 novembre 2013.

FrançoIs, Exhortation apostolique Amoris laetetia sur l'amour dans la famille, Rome, 19 mars 2016.

LEgRAND, Hervé. « Le développement d)Églises-sujets une requête de Vatican II », in Giuseppe Alberigo dir. Les Églises après Vatican II: dynamisme et prospective. Paris: Beauchesnes, coll. Théologie historique 61, 1981, pp. 149-184.

Routhier, Gilles. « Le Sensus fidei. L'interrelation entre tous les membres du peuple de Dieu » dans Timothy Radcliffe et Gilles Routhier Vox populi, vox Dei ?. Paris: Médiaspaul, 2016, pp. 41-76. 
Theobald, Christoph. « L'Exhortation apostolique Evangelii gaudium. Esquisse d'une interprétation originale du Concile Vatican II », dans Revue théologique de Louvain 46 (2015), pp. 321-340.

THILs, Gustave. « La communauté ecclésiale sujet d〉action et sujet de droit ». Revue Théologique de Louvain, 4 (1973), pp. 443-468.

\section{Gilles Routhier}

Docteur en Thélogie - Université Catholique de Paris Docteur en Histoire des Religions et Anthropologie religieuse Université de Paris IV (Sorbonne) Université Laval (Québec) Québec - Canada E-mail: Gilles.Routhier@ftsr.ulaval.ca

Recebido em: 20/09/16 Aprovado em: 23/09/16 